\title{
Températures et varicocèles
}

\author{
Roger MIEUSSET
}

Centre de Stérilité Masculine et Groupe de Recherche en Fertilité Humaine CHU Toulouse, France

\section{RESUME}

Bien que retrouvée chez des hommes féconds, la varicocèle est considérée comme responsable de l'infécondité par de nombreux auteurs. L'"hyperthermie' est l'un des mécanismes physiopathologiques évoqués pour expliquer les altérations de la spermatogenèse dues à la varicocèle. A partir des résultats rapportés dans la littérature concernant à la fois des situations cliniques chez l'homme et des données expérimentales chez l'animal, les constatations suivantes peuvent être faites : dans les populations d'hommes porteurs de varicocèle unilatérale, les températures moyennes testiculaires et scrotales sont augmentées de façon bilatérale dans la plupart des études. La bilatéralité de la varicocèle n'est pas associée à une augmentation plus importante de la température. L'effet de la cure chirurgicale sur la température testiculaire ou scrotale reste discuté. Enfin, le modèle animal de varicocèle expérimentale n'a pas jusqu'alors apporté des données supplémentaires concernant les mécanismes par lesquels la température testiculaire est augmentée en cas de varicocèle. En conclusion, s'il existe bien une association entre varicocèle et température testiculaire, cette dernière n'est pas systématiquement augmentée.

Mots clés. Infécondité, varicocèle, température, débit sanguin, homme, animal

\section{INTRODUCTION}

L'infécondité masculine peut résulter de causes très différentes ; pour certaines pathologies, comme par exemple « la varicocèle ", la certitude de causalité n'est pas encore clairement établie. La varicocèle est une varice localisée, au niveau du cordon spermatique, sur le trajet des veines drainant le sang de retour du testicule. Cette varice est retrouvée lors de l'examen clinique chez environ $12 \%$ des hommes dans la population générale et chez environ $25 \%$ chez les hommes consultant pour une infécondité. Les raisons de l'existence d'une varicocèle, qui survient naturellement dans d'autres espèces à scrotum pendulaire comme le bélier [6], ne sont pas connues chez l'homme. Lorsqu'elle est présente chez un homme infécond, la varicocèle est considérée, à partir de critères variés et non toujours précisément définis, comme la cause de cette infécondité. En vertu de quoi, le traitement étiologique de cette infécondité repose sur l'exérèse chirurgicale de la varicocèle ou son occlusion [47]. La pathogénicité de la varicocèle sur la fertilité, c'est à dire les mécanismes par lesquels la varice va réduire la capacité de l'homme à faire un enfant, est expliquée actuellement par plusieurs hypothèses [39] parmi lesquelles "l'hyperthermie » ou augmentation anormale de la température locale.

Nous allons faire l'état de la question sur cette hypothèse thermique des effets de la varicocèle sur la fertilité masculine, en prenant en compte les données cliniques chez l'homme et les données expérimentales chez l'animal.

\section{Correspondance :}

Mieusset Roger. Centre de Stérilité Masculine, Hôpital La Grave, La Grave 31052 Toulouse cedex, France

e-mail : mieusset.r@chu-toulouse.fr Communication au XVIII Congrès de la Société d'Andrologie de Langue Française, Montpellier, 13-15 décembre 2001. 


\section{DONNEES HUMAINES}

Il existe 25 études dans la littérature faisant état de mesure de la température testiculaire ou scrotale chez les hommes porteurs de varicocèle. Dans huit de ces études, les auteurs ne rapportent aucune donnée chiffrée en ce qui concerne les températures mesurées, soit $[23,30,25,15]$ en raison de la technique de mesure utilisée (thermographie), soit malgré la technique de mesure $[5,11,12,22]$. Bien que toutes ces études aient rapporté une augmentation de la température en cas de varicocèle, elles sont exclues de la présente analyse pour absence de données chiffrées. Pour ce qui est des 17 études restantes, elles présentent des différences méthodologiques de deux types.

Populations étudiées. Le diagnostic de varicocèle repose toujours sur l'examen clinique, seul ou avec échographie doppler ; les patients sont soit des hommes fertiles, soit des hommes inféconds, soit des hommes inféconds avec paramètres spermatiques 'altérés'. L'effectif des populations étudiées est parfois insuffisant pour valider les faits observés, avec un nombre inférieur à 30 (sept études), voire à moins de dix (deux études).

L'établissement de la spécificité d'une caractéristique ('l'hyperthermie') liée à une atteinte (la varicocèle) susceptible d'entraîner une maladie (l'infertilité) repose en partie sur la comparaison avec une population de référence. Parce que la question posée est celle de la pathogénicité de la varicocèle sur la fertilité, cette population de référence doit être constituée d'hommes féconds. Trois études ne remplissent pas cette condition en raison d'un groupe témoin soit absent, soit constitué d'hommes inféconds sans varicocèle. Enfin, pour quatre autres études, le groupe témoin a un effectif inférieur à dix.

Conditions de la mesure de la température. Une anesthésie, par deux fois générale, a été pratiquée dans trois études. L'acclimatation à la température du lieu de mesure n'a pas été réalisée dans 3 études, n'est pas précisée dans 5, et s'étend sur une durée de 5 à 10 minutes dans 9 études. L'habillement des patients lors de la mesure reste non précisé dans 13 études.

Quant à la mesure de température, elle est faite au niveau du testicule dans 5 études et du scrotum dans 13 études. Sauf pour 2 études (enregistrement en continu), cette mesure est unique et réalisée au moyen de thermomètres électronique ( $\mathrm{n}=10$ études), à mercure $(\mathrm{n}=4)$, infra-rouge $(\mathrm{n}=$ $2)$, à flux thermique $(n=1)$, voire par thermographie microondes $(\mathrm{n}=1)$. La validation des moyens de mesure utilisés pour évaluer la température du testicule ou du scrotum n'est pas rapportée dans toutes ces études.

Ces différences méthodologiques étant précisées, nous allons aborder successivement les résultats des mesures de température en cas de varicocèle gauche, puis bilatérale pour ce qui est de la température des testicules puis du scrotum. Toutefois, pour des raisons didactiques, nous traiterons tout d'abord les quatre études qui ne rapportent aucune modification de température en cas de varicocèle, trois de ces études étant très fréquemment citées pour invalider un quelconque effet « hyperthermiant » de la varicocèle.

\section{Varicocèle et températures inchangées}

L'une des quatre études [1] est en fait un travail dans lequel l'auteur cherche à savoir si la cure chirurgicale de la varicocèle induit une modification de la température testiculaire dans une population d'hommes fertiles et inféconds sélectionnés sur la seule présence d'une varicocèle. Il n'existe pas de population de référence dans cette étude pour laquelle l'auteur conclut à l'absence d'effet thermique net de la varicocelectomie.

Pour deux des études [40, 37], la généralisation des résultats obtenus - température inchangée en cas de varicocèle est à pondérer en raison d'une part de la faiblesse des effectifs et, d'autre part, de l'existence d'une anesthésie, cette dernière étant responsable de modifications importantes de la thermorégulation, comme cela a été démontré chez l'animal [4].

Enfin, dans une étude récente [27] les auteurs ne retrouvent aucune différence des températures moyennes scrotales entre porteurs et non porteurs de varicocèle. Plusieurs aspects méthodologiques jètent cependant un doute sur la validité des résultats. En effet, les deux populations présentent des biais de sélection importants puisque, pour un âge de 20 ans (médiane), un tiers des hommes sans varicocèle (population de référence) et trois quart des hommes avec varicocèle (population étudiée) présentent des altérations du sperme alors qu'aucun de ces hommes n'est infécond. De plus, les auteurs retrouvent bien une augmentation significative de la température scrotale moyenne gauche par rapport à la droite dans la population des hommes avec varicocèle.

\section{Températures testiculaires et varicocèle gauche}

Indépendamment de la technique de mesure $[8,7]$, une varicocèle gauche est associée à une augmentation de la température testiculaire moyenne qui est bilatérale et symétrique (Tableau 1) par comparaison à une population témoin. Toutefois, l'étude la plus récente [7] rapporte aussi une augmentation bilatérale et symétrique de la température testiculaire moyenne dans une population d'hommes inféconds non porteurs de varicocèle par rapport à une population témoin, augmentation dont l'intensité semble moindre que celle observée en cas de varicocèle (Tableau 1). Les résultats de cette dernière étude confirment a posteriori ceux précédemment rapportés dans une autre étude [46] qui trouvait une augmentation bilatérale et symétrique de la température testiculaire moyenne en cas de varicocèle par comparai- 
son avec un groupe d'hommes inféconds sans varicocèle (Tableau 1).

En résumé, dans des populations d'hommes inféconds porteurs d'une varicocèle gauche, il existe une augmentation bilatérale et symétrique des températures testiculaires moyennes (par rapport à la population témoin). Toutefois cette augmentation est aussi retrouvée dans une population d'hommes inféconds sans varicocèle.

\section{Températures scrotales et varicocèle gauche}

Onze études $[20,38]$ rapportent les résultats de mesures de la température scrotale chez des hommes inféconds avec varicocèle gauche ainsi que dans des populations de référence constituées d'hommes fertiles ou féconds sans varicocèle. Des neuf études dans lesquelles les températures scrotales ont été mesurées des deux côtés [48, 50, 28, 49, 8, $2,31,24,45]$, il apparaît une augmentation des températures scrotales moyennes droite et gauche par rapport à la population témoin. Pour quatre de ces études [28, 2, 31, 24], l'augmentation est par ailleurs significativement plus importante du côté de la varicocèle que du côté opposé (Tableau 2).

En résumé, dans les populations d'hommes inféconds porteurs d'une varicocèle gauche, les températures scrotales moyennes droite et gauche sont augmentées.

\section{Températures scrotales et varicocèle bilatérale}

Par rapport à des populations témoins, les hommes inféconds porteurs d'une varicocèle bilatérale présentent une augmentation bilatérale des températures scrotales moyennes droite et gauche (Tableau 3), indépendamment de la technique de mesure. L'intensité de cette augmentation n'est toutefois pas différente de celle observée en cas de varicocèle gauche $[49,45]$.

En résumé, dans les populations d'hommes inféconds avec varicocèle bilatérale, les températures scrotales moyennes droite et gauche sont augmentées; l'intensité de cette augmentation est similaire à celle observée en cas de varicocèle unilatérale.

\section{Températures scrotales, varicocèles et absence de varicocèle}

Trois études réalisant des mesures de la températures scrotales chez des hommes inféconds porteurs de varicocèle comprennent, outre une population témoin constituée d'hommes féconds, une population d'hommes inféconds sans varicocèle (Tableau 4). Par rapport aux populations témoins, ces études rapportent une augmentation des températures scrotales moyennes droite et gauche dans les populations infécondes, que les patients soient porteurs ou non d'une varicocèle [28, 49]. L'une des trois études [2] ne retrouve aucune modification des températures scrotales moyennes dans la population inféconde sans varicocèle ; des facteurs peuvent, toutefois, être source de biais dans cette étude, tels que la réalisation des mesures en position debout, sans acclimatation à la température ambiante chez des patients non dévêtus.

En résumé, dans des populations d'hommes inféconds, qu'ils soient porteurs ou non d'une varicocèle, il existe une augmentation bilatérale et symétrique des températures scrotales moyennes.

\section{Températures et cure chirurgicale de la varicocèle}

Quatre [1, 46, 24, 45] des études ayant réalisé des mesures de température locale avant et après cure chirurgicale d'une varicocèle uni ou bilatérale chez les mêmes patients rapportent des données chiffrées (Tableau 5). La plus ancienne [1], qui présente des problèmes méthodologiques déjà évoqués, ne retrouve aucune différence entre les températures testiculaires moyennes droite et gauche après chirurgie par rapport à avant. Cependant, une ré-analyse des données individuelles [29] avait permis de montrer qu'il existait dans un sous-groupe oligospermique de ces patients une relation entre température scrotale et production de spermatozoïdes. Dans l'étude de Yamaguchi et al. [46], sans groupe témoin, la varicocelectomie est associée à une réduction bilatérale de la température testiculaire moyenne, mais cette réduction n'est significative que du côté de la varice.

En ce qui concerne la température scrotale, la moyenne des multiples mesures réalisées en continu pendant la journée ne montre pas de réduction significative des températures scrotales moyennes droite et gauche après la cure chirurgicale [24]. A l'opposé, dans l'étude la plus récente [45], les températures scrotales moyennes droite et gauche sont significativement moindres après chirurgie, et non différentes de celles observées dans une population témoin en cas de varicocèle gauche. Cette réduction est aussi observée après cure chirurgicale d'une varicocèle bilatérale [45], mais les températures ne retrouvent pas des valeurs identiques à celles observées dans la population témoin (Tableau 5).

En résumé, la littérature présente des résultats divergents en ce qui concerne l'effet de la cure chirurgicale de la varicocèle sur les températures moyennes testiculaires ou scrotales. En l'état actuel, les données sont insuffisantes pour conclure.

En synthèse des données cliniques rapportées chez l'homme, les populations de patients porteurs d'une varicocèle présentent une augmentation des températures moyennes droite et gauche testiculaires $[8,46,7]$ et scrotales $[48,50$, $28,49,8,2,31,24,45]$, que la varicocèle soit uni ou bilatérale. Il apparaît donc tentant de déduire de ces données que la varicocèle agirait sur la fertilité par l'intermédiaire d'une augmentation de la température testiculaire. Cette affirmation est toutefois non recevable sous cette formulation chez l'homme, entre autre pour les raisons suivantes :

l'augmentation rapportée ne concerne que les températures 
Tableau I : Températures testiculaires et varicocèle gauche.

\begin{tabular}{|c|c|c|c|c|}
\hline \multirow[t]{2}{*}{ Auteurs(Moyen de mesure) } & \multirow{2}{*}{$\begin{array}{l}\text { Populations } \\
\text { étudiées }\end{array}$} & \multirow{2}{*}{$\begin{array}{l}\text { Populations } \\
\text { de référence }\end{array}$} & \multicolumn{2}{|c|}{ Différence $\left({ }^{\circ} \mathrm{C}\right)$ de température* } \\
\hline & & & Droit & Gauche \\
\hline $\begin{array}{l}\text { Goldstein \& Eid, } 1989 \text { [8] } \\
\text { (Thermomètre électronique) }\end{array}$ & $\begin{array}{l}\text { - Varicocèle } \mathrm{G} \\
(\mathrm{n}=26)\end{array}$ & $\begin{array}{l}\text { - Témoins } \\
(\mathrm{n}=45)\end{array}$ & $+2,4$ & $+2,7$ \\
\hline Yamaguchi et al, 1989 [46] & $\begin{array}{l}\text { - Varicocèle } G \\
(\mathrm{n}=89)\end{array}$ & $\begin{array}{l}\text { - Témoins } \\
(\mathrm{n}=0)\end{array}$ & - & - \\
\hline (Thermomètre à flux) & & $\begin{array}{l}\text { - Inféconds sans } \\
\text { varicocèle }(n=66)\end{array}$ & $+0,25$ & $+0,22$ \\
\hline Gazvani et al, 2000 [7] & $\begin{array}{l}\text { - Varicocèle } \mathrm{G} \\
(\mathrm{n}=38)\end{array}$ & $\begin{array}{l}\text { - Témoins } \\
(\mathrm{n}=20)\end{array}$ & $+1,1$ & $+1,1$ \\
\hline (Thermographie micro-ondes) & $\begin{array}{l}\text { Inféconds sans } \\
\text { varicocèle }(n=52)\end{array}$ & & $+0,6$ & $+0,6$ \\
\hline
\end{tabular}

* Toutes les valeurs sont significativement différentes de celles des populations auxquelles elles sont comparées.

Tableau 2 : Températures scrotales et varicocèle gauche

\begin{tabular}{|c|c|c|c|c|}
\hline \multirow[t]{2}{*}{ Auteurs } & \multirow[t]{2}{*}{$\begin{array}{l}\text { Témoins } \\
\text { n }\end{array}$} & \multirow[t]{2}{*}{$\begin{array}{l}\text { Varicocèle } \\
\text { gauche n }\end{array}$} & \multicolumn{2}{|c|}{$\begin{array}{l}\text { Différence de température } * \\
\text { par rapport aux témoins }\left({ }^{\circ} \mathrm{C}\right)\end{array}$} \\
\hline & & & Droit & Gauche \\
\hline Zorgniotti, [48] & 35 & 50 & $+0,7$ & $+0,6$ \\
\hline Mieusset, [28] & 37 & 49 & $+0,3$ & $+0,6 * *$ \\
\hline Zorgniotti, [49] & 30 & 76 & $+1,3$ & $+1,4$ \\
\hline Ali, [2] & 17 & 23 & $+1,1$ & $+1,6 * *$ \\
\hline Zorgniotti, [50] & 11 & 7 & $+1,3$ & $+1,0$ \\
\hline Takada, [38] & 10 & 20 & ND & $+1,7$ \\
\hline Jockenhövel, [20] & 8 & 28 & ND & $+1,2$ \\
\hline Lerchl, [24] & 6 & 48 & $+0,6$ & $+1,0 * *$ \\
\hline Goldstein, [8] & 45 & 26 & $+2,8$ & $+2,4$ \\
\hline Saliz, [31] & 9 & 58 & $+0,7$ & $+1,0 * *$ \\
\hline Wright, [45] & 45 & 30 & $+1,0$ & $+1,5$ \\
\hline
\end{tabular}

* Toutes les valeurs sont significativement différentes de celles observées dans la population témoin correspondante.

** Différence significative entre coté droit et gauche dans la population avec varicocèle.

Tableau 3 : Températures scrotales et varicocèle bilatérale

\begin{tabular}{|c|c|c|c|c|}
\hline \multirow[t]{2}{*}{ Auteurs (thermomètre) } & \multirow[t]{2}{*}{$\begin{array}{l}\text { Témoins } \\
\text { n }\end{array}$} & \multirow{2}{*}{$\begin{array}{l}\text { Varicocèle } \\
\mathbf{G}=\text { gauche } \\
\mathbf{B}=\text { bilatérale } \mathbf{n}\end{array}$} & \multicolumn{2}{|c|}{$\begin{array}{l}\text { Différence de température } * \\
\text { par rapport aux témoins }\left({ }^{\circ} \mathrm{C}\right)\end{array}$} \\
\hline & & & Droit & Gauche \\
\hline Zorgniotti \& Sealfon, 1988 [49] (à mercure) & 30 & $\begin{array}{l}G: n=76 \\
B: n=32\end{array}$ & $\begin{array}{l}+1,3 \\
+1,3\end{array}$ & $\begin{array}{l}+1,4 \\
+1,4\end{array}$ \\
\hline Wright et al, 1997 [45] (électronique) & 45 & $\begin{array}{l}G: n=30 \\
B: n=89\end{array}$ & $\begin{array}{l}+1,0 \\
+1,0\end{array}$ & $\begin{array}{l}+1,5 \\
+1,5\end{array}$ \\
\hline
\end{tabular}

* Toutes les valeurs sont significativement différentes de celles observées dans la population témoin correspondante. 
Tableau 4 : Températures scrotales, varicocèles uni ou bilatérale, absence de varicocèle.

\begin{tabular}{|c|c|c|c|c|}
\hline \multirow[t]{2}{*}{ Auteurs (thermomère) } & \multirow[t]{2}{*}{$\begin{array}{l}\text { Témoins } \\
\text { n }\end{array}$} & \multirow{2}{*}{$\begin{array}{l}\text { Varicocèles } \\
\text { VG = gauche } \\
\text { VB = bilatérale } \\
\text { VA = absente }\end{array}$} & \multicolumn{2}{|c|}{$\begin{array}{l}\text { Différence de température } \\
\text { par rapport aux témoins }\left({ }^{\circ} \mathrm{C}\right)\end{array}$} \\
\hline & & & Droit & Gauche \\
\hline $\begin{array}{l}\text { Mieusset et al, } 1987 \text { [28] } \\
\text { (à mercure) }\end{array}$ & 37 & $\begin{array}{l}V G: n=49 \\
V A: n=101\end{array}$ & $\begin{array}{l}+0,3 \\
+0,4\end{array}$ & $\begin{array}{l}+0,6 \\
+0,4\end{array}$ \\
\hline $\begin{array}{l}\text { Zorgniotti \& Sealfon, } 1988 \text { [49] } \\
\text { (à mercure) }\end{array}$ & 30 & $\begin{array}{l}V G: n=76 \\
V B: n=32 \\
V A: n=86\end{array}$ & $\begin{array}{l}+1,3 \\
+1,3 \\
+1,3\end{array}$ & $\begin{array}{l}+1,4 \\
+1,4 \\
+1,4\end{array}$ \\
\hline $\begin{array}{l}\text { Ali et al, } 1990[2] \\
\text { (à mercure) }\end{array}$ & 17 & $\begin{array}{l}V G: n=23 \\
V A: n=32\end{array}$ & $\begin{array}{l}+1,1 \\
+0,0\end{array}$ & $\begin{array}{l}+1,6 \\
+0,1 *\end{array}$ \\
\hline
\end{tabular}

* Sauf ces valeurs, toutes sont significativement différentes de celles observées dans la population témoin correspondante.

Tableau 5 : Températures testiculaires ou scrotales et varicocelectomie

\begin{tabular}{|c|c|c|c|}
\hline Auteurs (Conditions *) & $\begin{array}{l}\text { Différence de température }\left({ }^{\circ} \mathrm{C}\right) \text { : } \\
\text { après/avant chirurgie }\end{array}$ & $\begin{array}{l}\text { Effet de la chirurgie } \\
\text { sur la température }\end{array}$ & $\begin{array}{l}\text { Différence de } \\
\text { température }\left({ }^{\circ} \mathrm{C}\right): \\
\text { après chirurgie /témoins }\end{array}$ \\
\hline $\begin{array}{l}\text { Agger } 1971 \text { [1] } \\
\text { (Testis, th. elect., all.,) }\end{array}$ & $\begin{array}{l}\mathrm{D}^{* *}:+0,12 \\
\mathrm{G}:-0,01\end{array}$ & $\begin{array}{l}\text { non } \\
\text { non }\end{array}$ & $\begin{array}{l}\text { pas de } \\
\text { groupe témoin }\end{array}$ \\
\hline $\begin{array}{l}\text { Yamaguchi et al, } 1989 \text { [46] } \\
\text { (Testis, th. à flux, all., acclim.) }\end{array}$ & $\begin{array}{l}D:-0,25 \\
\text { G : }-0,33\end{array}$ & $\begin{array}{l}\text { non } \\
\text { oui }\end{array}$ & $\begin{array}{l}\text { pas de } \\
\text { groupe témoin }\end{array}$ \\
\hline $\begin{array}{l}\text { Lerchl et al, } 1993 \text { [24] } \\
\text { (Scrotum, th. elect., moy. jour.) }\end{array}$ & $\begin{array}{l}\text { D : }-0,11 \\
\text { G : }-0,20\end{array}$ & $\begin{array}{l}\text { non } \\
\text { non }\end{array}$ & $\begin{array}{l}+0,76 \\
+0,64\end{array}$ \\
\hline $\begin{array}{l}\text { Wright et al, } 1997 \text { [45] } \\
\text { (Scrotum, th. elect., all., acclim.) }\end{array}$ & $\begin{array}{l}\text { D : }-0,97 \\
\text { G }:-1,63 \\
\text { BD : }-0,80 \\
\text { BG : }-0,80\end{array}$ & $\begin{array}{l}\text { oui } \\
\text { oui } \\
\text { oui } \\
\text { oui }\end{array}$ & $\begin{array}{c}-0,01 \\
-0,02 \\
+0,24 \\
+0,68\end{array}$ \\
\hline
\end{tabular}

* Testis, scrotum = températures testiculaires, scrotales ; th.= thermomètre ; elect. = électronique ; all. : mesure en position allongée ; acclim. : acclimatation à la température du lieu de mesure ; moy.jour. = moyenne des mesures enregistrées en continu pendant le jour.

** $\mathrm{D} ; \mathrm{G}=$ température moyenne droite, gauche en cas de cure chirurgicale d'une varicocèle gauche $; \mathrm{BD}, \mathrm{BG}=$ température moyenne droite, gauche en cas de cure chirurgicale d'une varicocèle bilatérale.

moyennes, ce qui laisse entendre, comme le montre les données individuelles, que la température n'est pas systématiquement augmentée en présence d'une varicocèle. En effet, chez des patients inféconds avec varicocèle gauche, la température scrotale n'est augmentée que chez un homme sur deux, que la meure soit faite par thermomètrie à mercure [28] ou thermographie de contact [15]. Il pourrait donc exister au moins deux types de varicocèles, selon la présence ou l'absence d'une élévation anormale de la température.

Chez les hommes inféconds porteurs d'une varicocèle, l'existence d'une température scrotale anormalement augmentée, augmentation dont les effets sur la spermatogenèse et la fertilité sont bien documentés chez l'homme [29, 33], signifie-t-elle pour autant que la pathogénicité de la varicocèle s'exerce par l'augmentation de la température ? Aucune donnée humaine clinique ou expérimentale ne permet actuellement de l'affirmer. Plus encore, il existe des données autorisant une autre interprétation : en effet, l'existence de températures scrotales ou testiculaires moyennes anormalement élevées chez les hommes inféconds sans varicocèle $[28,49,7]$ indique que l'augmentation de température constatée en présence d'une varicocèle pourrait 
non seulement ne pas être spécifique de la varicocèle, mais aussi ne pas résulter de la présence de la varicocèle. A cette étape de la problématique, les données expérimentales obtenues chez l'animal peuvent elles apporter une solution?

\section{DONNEES ANIMALES}

Une quarantaine de publications font état de l'induction par voie chirurgicale d'une varicocèle gauche expérimentale (VGE). Quinze de ces publications rapportent les températures testiculaires (moyennes) mesurées sous anesthésie générale chez des animaux avant et après VGE ainsi que chez des témoins. Quatre familles animales ont été utilisées : le rat ( 3 espèces ; 11 études), le singe ( 2 espèces ; 3 études), le chien et le lapin (une étude pour chacune). Dans tous les cas, la VGE fut induite par la ligature partielle (50 à $80 \%$ de réduction) de la veine rénale gauche (ou de son équivalent) associée à une ligature plus ou moins complète des autres voies veineuses efférentes du testicule ; cette technique entraîne chez environ $80 \%$ des animaux une dilatation de la veine spermatique gauche, c'est à dire une varicocèle gauche. Cette varicocèle induite chez l'animal est similaire à celle survenant chez l'homme [42].

Les températures testiculaires moyennes droite et gauche sont significativement augmentées dans la population d'animaux après création d'une VGE, par rapport aux animaux témoins, que ce soit chez le rat $[32,9,10,18,19,34$, $43,44,16,17]$, le singe $[21,13,14]$, le chien [32] ou le lapin $[35,36]$. Cette augmentation de la température testiculaire moyenne n'est pas un phénomène transitoire puisqu'elle est toujours présente 3 ou 6 mois après la création d'une VGE chez le rat $[18,17]$, le chien [32] ou le lapin [35], voire 3 ans chez le singe [13, 14]. Deux arguments font que cette augmentation des températures moyennes testiculaires est bien une conséquence de la création d'une VGE :

a) les populations d'animaux sham-opérés (i.e. ayant subi toutes les procédures appliquées aux animaux ayant eu une VGE à l'exception de la ligature de la veine rénale) n'ont jamais d'augmentation des températures testiculaires moyennes, quels que soient l'espèce considérée et le temps écoulé depuis la chirurgie.

b) la suppression, par une varicocelectomie comme pratiquée chez l'homme, de la VGE est associée à un retour des températures testiculaires moyennes à des valeurs normales, i.e. similaires à celles des populations témoins, que la varicocelectomie ait été réalisée 1 mois ou 3 mois après la création de la VGE chez le rat ou le lapin [18, 35], ou 14 mois après VGE chez le singe [13].

En résumé, les études montrent chez différentes espèces animales que la création chirurgicale d'une varicocèle gauche est associée à une augmentation des températures testiculaires moyennes droite et gauche dans la population opérée par rapport à une population témoin. Cette augmentation perdure dans le temps, mais disparaît après correction chirurgicale de la varicocèle induite expérimentalement.

Certains auteurs ont essayé d'expliquer les mécanismes par lesquels ces températures testiculaires moyennes droite et gauche étaient augmentées après VGE, le plus fréquemment cité étant une modification du débit sanguin testiculaire (DST) total. En effet, une VGE entraîne, outre une augmentation bilatérale et symétrique des températures testiculaires moyennes, un accroissement de 30 à $50 \%$ du DST total moyen (mesuré in vitro) des testicules droits et gauches, 1 mois $[32,9,19,43]$ ou 3 mois [18] après VGE chez le rat, ainsi que 3 mois après VGE chez le chien [32]. De plus, la correction chirurgicale de la VGE normalise à la fois le DST total moyen des testicules droits et gauches et les températures testiculaires moyennes droites et gauches chez le rat $[9,18]$.

En résumé, plusieurs auteurs ont montré que la création d'une VGE dans une population animale induisait une augmentation du débit sanguin total moyen des testicules droits et gauches, augmentation qui disparaît après correction chirurgicale de la VGE.

A partir de la co-existence de ces deux modifications associées à l'induction d'une VGE (augmentation bilatérale du DST total moyen et augmentation bilatérale de la température testiculaire moyenne), des auteurs ont suggeré que l'augmentation de température était la conséquence de l'augmentation du débit sanguin des testicules [32, 9]. La cascade physiopathologique suivante aurait donc lieu : induction d'une VGE, augmentation bilatérale du DST total, augmentation bilatérale de la température des testicules, cette dernière étant responsable des altérations de la spermatogenèse. Cependant, d'autres résultats rapportés chez l'animal font discuter le bien-fondé de cette explication physiopathologique des effets de la varicocèle sur la fertilité. Ces données contradictoires peuvent être synthétisées sous forme de trois questions :

1. Une VGE entraîne-t-elle toujours une augmentation du débit sanguin testiculaire? Cette formulation ne vise pas à remettre en question la validité des résultats rapportés par les auteurs précités, mais elle attire l'attention sur la méthodologie utilisée pour mesurer le débit sanguin des testicules [41]. D'autres auteurs ont en effet montré qu'une mesure du DST total in vivo chez le singe 5 mois après l'induction d'une VGE révélait une réduction de $20 \%$ à droite et de $50 \%$ à gauche du DST total moyen [13]. Avec cette même technique de mesure in vivo, une étude récente [26] rapporte une diminution de $20 \%$ du DST total moyen 30 minutes et un mois après induction d'une VGE chez le rat. 
Il n'est donc pas prouvé de façon certaine que l'induction d'une varicocèle chez l'animal soit associée à une augmentation du DST total.

2. Une augmentation de la température testiculaire peut-elle avoir pour origine d'autres facteurs que l'augmentation du débit sanguin testiculaire? La réponse est positive ; par exemple, l'adrénalectomie unilatérale gauche chez un groupe de rats [43] est associée à une augmentation bilatérale de la température testiculaire moyenne comme dans le cas de la VGE - sans aucune modification du DST total mesuré in vitro.

L'augmentation de la température testiculaire moyenne bilatérale peut donc résulter d'une altération unilatérale indépendamment de toute modification du DST total testiculaire.

3. Comment la VGE est-elle la cause des modifications observées du DST et de la température testiculaire ? Il parait bien difficile de répondre à cette question dans le modèle animal, même si l'on a précédemment rapporté que la varicocelectomie était associée à une normalisation des températures et des débits sanguins testiculaires moyens. En effet, la sympathectomie lombaire unilatérale gauche réalisée en l'absence de toute varicocèle chez un groupe de rats [10] est associée à une augmentation bilatérale du DST moyen (mesuré in vitro) et à une augmentation bilatérale des températures testiculaires moyennes.

L'augmentation bilatérale du débit sanguin et de la température testiculaire ne sont pas pathognomoniques de la varicocèle induite expérimentalement.

En conclusion de ces données cliniques chez l'homme et expérimentales chez l'animal, il semble bien qu'il existe une relation entre certaines varicocèles (lesquelles ?) et la température testiculaire ou scrotale. Mais il apparaît aussi que toutes les varicocèles ne sont pas associées à une augmentation anormale de cette température (si tant est que l'on se donne les moyens de définir la normalité de ladite température...). Des études sont encore nécessaire pour permettre d'identifier les varicocèles pathogènes pour la fertilité et les mécanismes de cette pathogénicité, que ce soit chez l'homme [3] ou chez l'animal [41]. De ces résultats découleront l'adéquation de la thérapeutique proposée.

\section{REFERENCES}

1. AGGER P. : Scrotal and testicular temperature : its relation to sperm count before and after operation for varicocele. Fertil. Steril., 1971, 22 : 286-296.

2. ALI J.I., WEAVER D.J., WEINSTEIN S., GRIMES E.M. Scrotal temperature and semen quality in men with and without varicocele. Arch. Androl., 1990, 24 : 215-219.

3. BENOFF S., GILBERT B.R. : Varicocele and male infertility. Hum. Reprod. Up., 2001, 7 : 47-54.
4. CAIRNIE A.B., LEACH K.E. : Quantitative study of cytological damage in mouse testis produced by exposure to heat. Can. J. Genet. Cytol., 1980, 22 :93-102.

5. DAVIDSON H.A. : Treatment of male subfertility. Testicular temperature and varicoceles. Practitionner, 1954, 173 : 703-708.

6. EZZI A., LADDS P.W., HOFFMANN D., FOSTER R.A., BRIGGS G.D. : Pathology of varicocele in the ram. Aust. Vet. J., 1988, $65: 11-15$

7. GAZVANI M.R., WOOD S.J., THOMSON A.J.M., KINGSLAND C.R., LEWIS-JONES D.I. : Assessement of testicular core temperatures using microwawe thermography. Hum. Reprod., 2000, $15:$ 1723-1726.

8. GOLDSTEIN M., EID J.F. Elevation of intratesticular and scrotal skin surface temperature in men with varicocele. J. Urol., 1989, $142: 743-745$.

9. GREEN K.F., TURNER T.T., HOWARDS S.S. : Varicocele : reversal of the testicular blood flow and temperature effects by varicocele repair. J. Urol., 1984, $131: 1208-1211$.

10. GREEN K.F., TURNER T.T., HOWARDS S.S. : Effects of varicocele after unilateral orchiectomy and sympathectomy. J. Urol., 1985, $134: 378-383$.

11. HANLEY H.G. : Surgical correction of errors of temperature regulation. In : Proceedings of the Second World Congress on Fertility and Sterility, vol 2. New York, Paul B Hoeber Inc, 1956 : 953-964.

12. HANLEY H.G., HARRISON R.G. : The nature and surgical treatment of varicocele. Brit. J. Surg., 1962, $50: 64-67$.

13. HARRISON R.M., LEWIS R.W., ROBERTS J.A. : Pathophysiology of varicocele in nonhuman primates : long-term seminal and testicular changes. Fertil. Steril., 1986, $46: 500-510$.

14. HARRISON R.M., SMITH B.A., ROBERTS J.A. : Testicular temperatures measured by thermistor probe and contact thermography. Fertil. Steril., 1990, $54:$ 173-174.

15. HSIUNG R., NIEVA H., CLAVERT A. : Scrotal hyperthermia and varicocele. In : Zorgniotti A.W. ed. Temperature and Environmental effects on the testis. Advances in Experimental Medicine and Biology. Vol. 286. NewYork, Plenum Press, 1991 : 241-244.

16. HSU H.S., CHANG L.S., CHEN M.T., WEI Y.H. : Decreased blood flow and defective energy metabolism in the varicocelebearing testicles. Eur. Urol., 1994, 25 : 71-75.

17. HSU H.S., WEI Y.H., LI A.F., CHANG L.S., CHEN M.T. : Defective mitochondrial oxidative phosphorylation in varicocele-bearing testicles. Urol., 1995, $46: 545-549$.

18. HURT G.S., HOWARDS S.S., TURNER T.T. : Repair of experimental varicoceles in the rat : long-term effects of testicular blood flow and temperature and cauda epididymal sperm concentration and motility. J. Androl., 1986, 7 : 271-276.

19. HURT G.S., HOWARDS S.S., TURNER T.T. : The effects of unilateral experimental varicocele are not mediated through the ipsilateral testis. J. Androl., 1987, 8 : 403-407.

20. JOCKENHÖVEL F., GRÄWE A., NIESCHLAG E. : A portable digital data recorder for long term monitoring of scrotal temperature. Fertil. Steril., 1990, $54: 694-700$.

21. KAY R., ALEXANDER N.J., BAUGHAM W.H. : Induced varicoceles in rhesus monkeys. . Fertil. Steril., 1979, 31 : 195-199.

22. KITAYAMA T. Study on testicular temperature in man. Acta Urol. Japon., 1965, $11:$ 435-437. 
23. KORMANO M., KAHANPÄÄ K., SVINHUFVUD U., TÄHTI E. : Thermography of varicocele. Fertil. Steril.,1970, $21: 558-$ 564.

24. LERCHL A., KECK C., SPITERI-GRECH J., NIESCHLAG E. : Diurnal variations in scrotal temperature of normal men and patients with varicocele before and after treatment. Int. J. Androl., 1993, 16:195-200.

25. LEWIS R.W., HARRISON R.M. : Contact scrotal thermography. II Use in the infertile male. Fertil. Steril., 1980, $34: 259-263$.

26. LI H.K., DUBOCQ F., JIANG Y. et al. : Effect of surgicallyinduced varicocele on testicular blood flow and Sertoli cell function. Urol., 1999, 53 : 1258-1262.

27. LUND L., NIELSEN K. : Varicocele testis and testicular temperature. Brit. J. Urol., 1996, 78 : 113-115.

28. MIEUSSET R., BUJAN L., MONDINAT C., MANSAT A., PONTONNIER F., GRANDJEAN H. : Association of scrotal hyperthermia with impaired spermatogenesis in infertile men. Fertil. Steril., 1987, 48 : 1006-1011.

29. MIEUSSET R., BUJAN L. : Testicular heating and its possible contributions to male infertility : a review. Int. J. Androl., 1995, $18: 169-184$.

30. MONTEYNE R., COMHAIRE F. : The thermographic characteristics varicocele : an anlysis of 65 positve registrations. Brit. J. Urol. 1978, $50: 118-120$.

31. SALIZ J.A., KASS E.J., STEINERT B.W. : The significance of elevated scrotal temperature in adolescent with varicocele. In : Zorgniotti A.W. ed. Temperature and Environmental effects on the testis. Advances in Experimental Medicine and Biology. Vol. 286. NewYork, Plenum Press, 1991 : 245-251.

32. SAYPOL D.C., HOWARDS S.S., TURNER T.T., MILLER E.D. : The influence of surgically induced varicocele on testicular blood flow, temperature, and histology in adult rats and dogs. J. Clin. Invest., 1981, $68: 39-45$.

33. SETCHELL B.P. : Heat and the testis. J. Reprod. Fert., 1998, $114: 179-194$.

34. SHOOK T.E., NYBERG L.M., COLLINS B.S. et al. : Pathological and immunological effects of surgically-induced varicocele in juvenile and adult rats. Am. J. Reprod. Immunol., 1988, $17: 141-144$.

35. SOFIKITIS N., MIYAGAWA I. : Effects of surgical repair of experimental left varicocele on testicular temperature, spermatogenesis, sperm maturation, endocrine function, and fertility in rabbit. Arch. Androl., 1992b, $29:$ 163-175.

36. SOFIKITIS N., TAKAHASHI C., NAKAMURA I., HIRAKAWA S., MIYAGAWA I. : Surgical repair of secondary right varicocele in rats with primary left varicocele : effects on fertility, testicular temperature, spermatogenesis, and sperm maturation. Arch. Androl., 1992a, $28: 43-52$.

37. STEPHENSON J.D., O'SHAUGHNESSY E.J. : Hypospermia and its relationship to varicocele and intrascrotal temperature. Fertil. Steril., 1968, $19: 110-117$.

38. TAKADA T., KITAMURA M., MATSUMIYA K., et al. : Infrared thermometry for rapid, non invasive detection of reflux of spermatic vein in varicocele. J. Urol., 1996, $156: 1652-1654$.

39. TAKIHARA A., SAKATOKU J., COCKETT A.T.K. : The pathophysiology of varicocele in male infertility. Fertil. Steril., 1991, $55: 861-868$.

40. TESSLER A.N., KRAHN H.P. : Varicocele and testicular temperature. Fertil. Steril., 1966, $17: 201-203$.
41. TURNER T.T. : The study of varicocele through the use of animals models. Hum. Reprod. Up., 2001, 7 : 78-84.

42. TURNER T.T., HOWARDS S.S. : The venous anatomy of experimental left varicocele : comparison with naturally occuring left varicocele in the human. Fertil. Steril., 1994, 62 : 869-875.

43. TURNER T.T., LOPEZ T.J. : Effects of experimental varicocele require neither adrenal contribution nor venous reflux. J. Urol., 1989, $142: 1372-1375$.

44. TURNER T.T., LOPEZ T.J. : Testicular blood flow in peripubertal and older rats and investigation into the mechanism of the bilateral respose to the unilateral lesion. J. Urol., 1990, 144 : 1018-1021.

45. WRIGHT E.J., YOUNG G.P., GOLDSTEIN M. : Reduction in testicular temperature after varocelectomy in infertile men. Urol., 1997, $50:$ 257-259.

46. YAMAGUCHI M., SAKOTU J., TAKIHARA H. : The application of intrascrotal deep body temperature in measurement for the non invasive diagnosis of varicocele Fertil. Steril., 1989, 52 : 295-301.

47. ZINI L., RIGOT J.M., BALLEREAU C., DEHAENE J.L., LEMAITRE L., MAZEMAN E. : Apport de l'embolisation de la varicocèle chez 51 patients infertiles. Andrologie, 2001, 11 : 5660.

48. ZORGNIOTTI A.W., MCLEOD J. :' Studies in temperature, human semen quality and varicocele. Fertil. Steril., 1973, 24 : 854-863.

49. ZORGNIOTTI A.W., SEALFON A.I. : Measurement of intrascrotal temperature in normal and subfertile men. J. Reprod. Fert., $1988,82: 563-566$.

50. ZORGNIOTTI A.W., TOTH A., MCLEOD J. : Infrared thermometry for testicular temperature determination. Fertil. Steril., $1979,32: 347-348$.

\section{ABSTRACT}

Varicoceles and temperature.

Roger MIEUSSET

Varicocele is considered to be responsible for male infertility by several authors, while varicocele may also exist in fertile men. Hyperthermia or "raised" testicular temperature is one of the pathophysiological mechanisms proposed to explain impaired spermatogenesis when a varicocele is present in infertile men. The reported clinical data in humans and experimental results of surgically-induced varicocele in animals indicate the following findings. In most studies, mean testicular and scrotal temperatures are bilaterally increased in populations of men with unilateral left varicocele compared to control populations. Similar results are observed following surgical induction of experimental left varicocele in ani- 
mals.

In the case of bilateral varicocele, the increase in mean temperature is similar to that observed in unilateral varicocele. Data concerning the effects of varicocelectomy on testicular or scrotal temperatures are still inconclusive in humans because of the discordant results in the small number of studies dealing with this topic. However, experimental data indicate that varicocelectomy results in recovery of normal mean temperature in animals submitted to surgically-induced varicocele, but experimental animal models of varicocele have failed to provide any explanation for the increase in temperature.

In conclusion, although there is a relationship between varicocele and testicular temperature, testicular temperature is not increased in every case of varicocele.

Key Words: Varicocele, temperature, blood flow, humans, animals. 